\title{
Методы автоматизации проектирования в среде AutoCAD
}

\author{
П. Б. Ломовцев, С. В. Болтач, Н. Ф. Митрофанова, Б. Г. Шинко
}

Одесская национальная академия пищевых технологий, ул. Канатная, 112, Одесса, 65039, Украина

\begin{abstract}
Создание масштабных или узкоспецчиализированых проектов в среде AиtоCAD занимает много времени. Для сокращения времени проектирования и добавления функиионала используются различные методы автоматизации проектирования. Большинство из них, кроме знаний команд и интерфейса среды АитоСАD, требуют навыков программирования. В данной работе рассмотрены методы автоматизации проектирования для области холодильной техники, которые требуют только знаний работы в среде AиtоСАD. Проанализирован каждый из методов, приведены примеры их использования и применения. Указаны достоинства и недостатки.
\end{abstract}

Ключевые слова: Методы автоматизаџии проектирования; АитоСАD; Принципиальная схема; Блок; Библиотека элементов; Сценарий; Макрокоманда.

\section{Методи автоматизації проектування в середовищі AutoCAD}

\author{
П. Б. Ломовцев, С. В. Болтач, Н. Ф. Мітрофанова, Б. Г. Шинко
}

Одеська національна академія харчових технологій, вул. Канатна, 112, Одеса, 65039, Україна

\begin{abstract}
Створення масштабних або узкоспеціалізірованних проектів в середовищі AиtоCAD займає велику кількість часу. Для скорочення часу проектування та додавання функиіоналу використовуються різні методи автоматизації проектування. Більшість з них, крім знань команд і інтерфейсу середовища AutoCAD, вимагають навичок програмування. У даній роботі розглянуті методи автоматизації проектування для області холодильної техніки, які вимагають тільки знань роботи в середовищі AutoСAD. Проаналізовано кожен з методів, наведені приклади їх використання $і$ застосування. Вказані переваги і недоліки.
\end{abstract}

Ключові слова: Методи автоматизації проектування; АиtоСАD; Принципова схема; Блок; Бібліотека елементів; Сиенарій; Макрокоманда.

DOI: http://dx.doi.org/10.15673/ret.v53i4.711

(C) The Author(s) 2017. This article is an open access publication

This work is licensed under the Creative Commons Attribution 4.0 International License (CC BY) http://creativecommons.org/licenses/by/4.0/

\section{1 Введение}

Современная технология проектирования холодильной техники не обходится без применения компьютерных программ, позволяющих выполнять необходимые расчеты, анализировать полученные данные, создавать документацию проекта и т.п. И вряд ли найдется инженер, который не знает об одной из самых популярных программ оформления графической документации AutoCAD. Формат файла электронного чертежа $d w g$ этой программной среды проектирования стал де-факто стандартом во всем мире. В 2017 году исполняется 35 лет, как кампания Autodesk выпустила первую версию AutoCAD. Однако, несмотря на почтенный возраст для компьютерной программы, этот программный продукт является очень востребованным у специалистов благодаря своей универсальности применения и открытости архитектуры, легкой адаптации к требованиям пользователей. B AutoCAD 2018 (на данный момент послед- няя выпущенная версия) можно не только создавать чертежи и схемы, но также создавать 3D модели деталей и сборок, распечатывать их на 3D принтере, передавать в другие программы и получать из них необходимые данные проекта, осуществлять совместную работу группы проектировщиков и т.д.

Но, несмотря на все новшества последних версий, одними из существенных достоинств AutoCAD является возможность адаптации рабочего места проектировщика под конкретные задачи. Это существенно облегчает работу и позволяет сосредоточиться на задачах принятия решений, а выполнение многих рутинных операций передать среде AutoCAD [1].

В этой статье рассмотрим и проведем анализ методов автоматизации проектирования в среде AutoCAD на примере применения в области холодильной техники. Большинство из этих методов присутствовало в предыдущих версиях программы, но используются далеко не всеми пользователями среды AutoCAD. Объяс- 
няется это следующим - не всем пользователям это требуется (чаще всего из-за незнания про различные методы автоматизации), сложностью создания дополнительных приложений-модулей, требующих навыков программирования и т.п.

Все рассматриваемые методы можно условно разделить на две категории:

- без применения языков программирования, к которым относятся использование блоков, макрокоманд и сценариев, для пользователей, хорошо знающих команды и интерфейс среды AutoCAD;

- с применением языков программирования, как специализированных (AutoLISP, DCL, DIESEL и др.), так и универсальных (VBA, C ++, C\#), с использованием API для более подготовленных пользователей.

\section{2 Методы автоматизации проектирования}

Одним из наиболее простых методов автоматизации графических работ является использование блоков. Блок - сохраняемый объект, который имеет имя, атрибуты, базовую точку вставки, возможность масштабирования и поворота. В блок может быть включено любое количество примитивов чертежа, в том числе другие блоки, может быть как частью чертежа, так и отдельным элементом. По возможностям изменения блоки делятся на статические и динамические.

Для создания блока часто выбираются элементы, которые используются множество раз в неизменном виде либо с изменениями в расположении, масштабе, выбираются из таблицы размерного ряда и т.п. В случае с принципиальной схемой таким элементом может выступать условное обозначение на схеме. Для чертежей деталей и сборочных единиц блок может быть использован как элемент детали, деталь, конструкция и т.п. Из блоков часто формируют библиотеки элементов, а также группируют их в отдельные панели с библиотеками элементов [2, 3].

На вкладке Вставка (Insert) выбирается кнопка Создать блок (Create Block). При нажатии появляется окно определения блока. В поле ввода Имя (Name) записывается имя блока, панель Базовая точка (Base point) отвечает за базовую указание точки вставки блока, её можно выбрать с помощью кнопки Указать (Pick point) или задать координаты. Панель Объекть (Object) предполагает выбор объектов, которые образуют блок и их поведение после создания блока на чертеже: Ocmaвить (Retain) - сохранить в том же виде, как и до создания блока; Преобразовать в блок (Convert to block) - заменить на созданный блок; Удалить (Delete) - удалить исходные элементы после создания блока. После выбора элементов блока его пиктограмма появляется напротив поля имени. Панель Поведение (Behavior) определяет, будет ли блок аннотативным и можно ли его расчленять на элементы. В Настройки (Settings) указываются единицы размерности блока (по умолчанию - миллиметры). Гиперссылка (Hyperlink) это возможность создания гиперссылки. Поле Описание (Description) отвечает за дополнительное описание блока (рисунок 1).

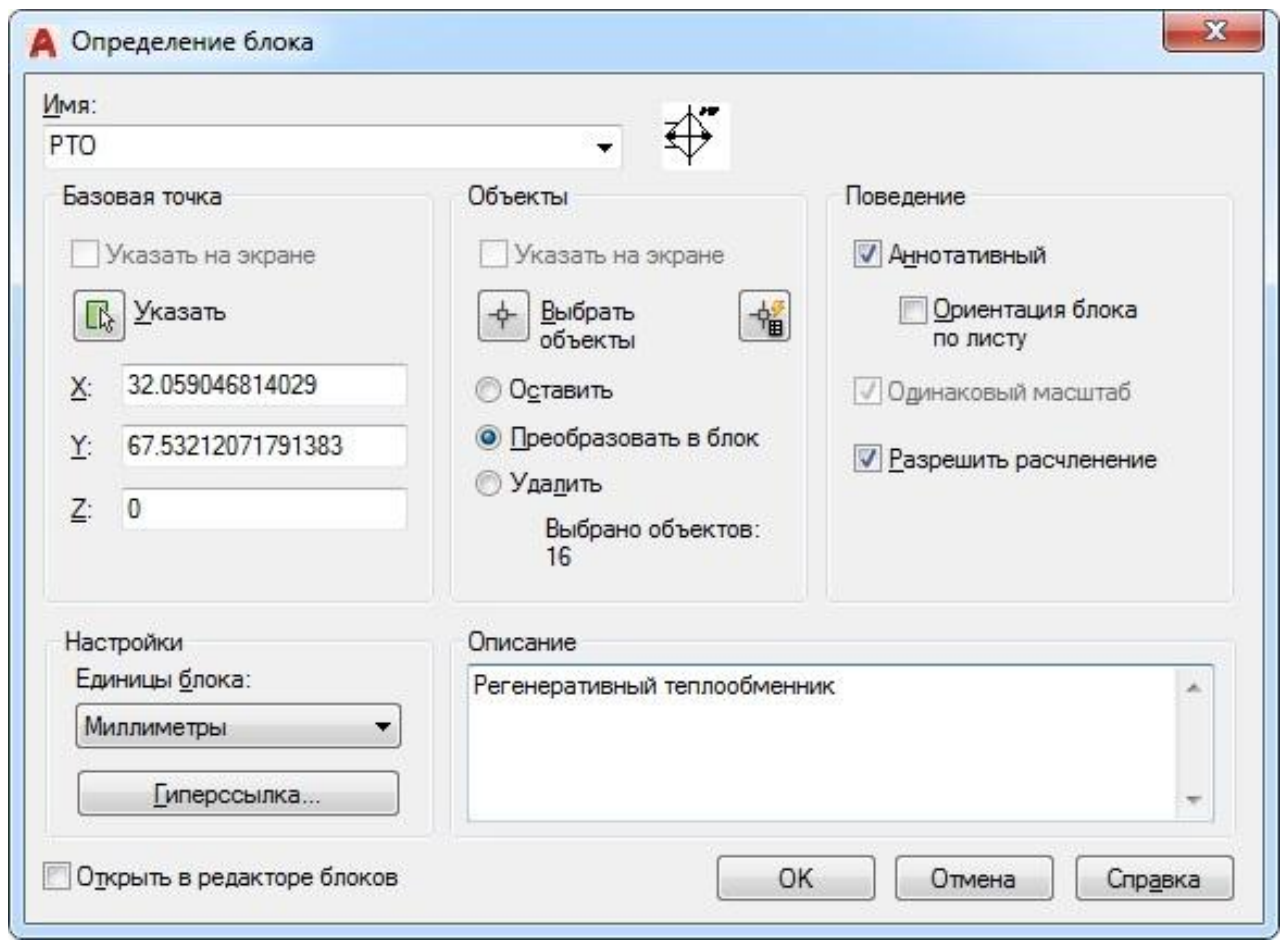

Рисунок 1 - Окно описания блока

Для вставки блока используется кнопка Вставка (Insert): выбирается сам блок и, с помощью перетягивания в нужное место на графическом поле либо задания координат размещения, располагается на чертеже.
При вставке блока таким образом он является статическим (создание и использование динамических блоков - тема для отдельной статьи). Пример вставки блока показан на рисунке 2. 


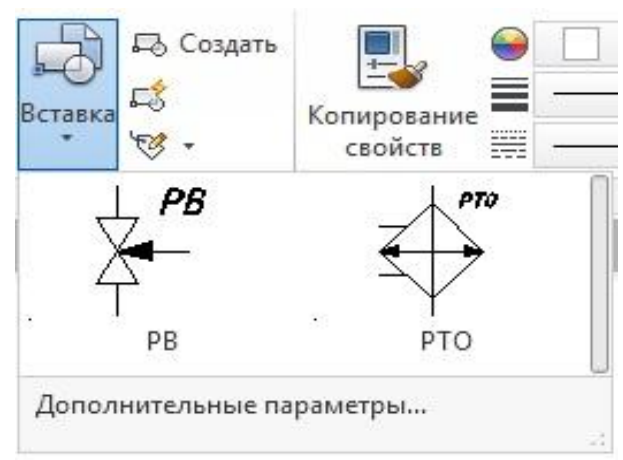

Рисунок 2 - Вставка блока

При выборе опции Дополнительные параметры (More Options) появляется диалоговое окно для вставки изменяемого блока (рисунок 3 ).

В окне Вставка (Insert) задаются: точка вставки, масштаб, угол поворота, единицы блока и возможность его расчленять сразу после вставки. Параметры можно задать сразу или в процессе вставки блока.

Примеры возможного использования: набор блоков с определёнными обозначениями для вентиляции, водоснабжения или канализации; блоки специальных обозначений для любых принципиальных схем; блоки типовых таблиц; часто используемые в чертеже детали и их обозначения и т.д. Использование статических блоков позволяет добавлять спроектированный единожды элемент необходимое количество раз, а динами- ческих ещё и изменять при этом необходимые размеры и располагать с нужным поворотом. Разница затраты времени с использованием этого метода автоматизации по сравнению с обычным копированием элементов при построении чертежей будет зависеть от количества использованных блоков и их сложности. При необходимости корректировки элемента редактируется только один экземпляр блока, а все остальные вхождения блока обновляются автоматически, что существенно снижает количество ошибок на чертеже.

Преимущества использования: легкость формирования блока, доступность способа, возможность изменения блока, контроль всех вхождений блоков, сохранение блока отдельным файлом, повышение производительности.

Недостатки использования: изначально необходимо создать блок или подключить библиотеку готовых элементов.

Вторым методом непрограммируемой автоматизации в среде AutoCAD по частоте использования являются макрокоманды. Макрокоманда - это ряд команд либо одна команда AutoCAD, с указанными свойствами, которые могут быть записаны в определённой последовательности и запускаются с одной созданной кнопки. Для чистого использования макрокоманд нет необходимости изучать языки программирования, необходимо знать только сами команды, их свойства, правила записи и спецсимволы макрокоманд. Подробно информация о специальных символах представлена в $[4,5]$.

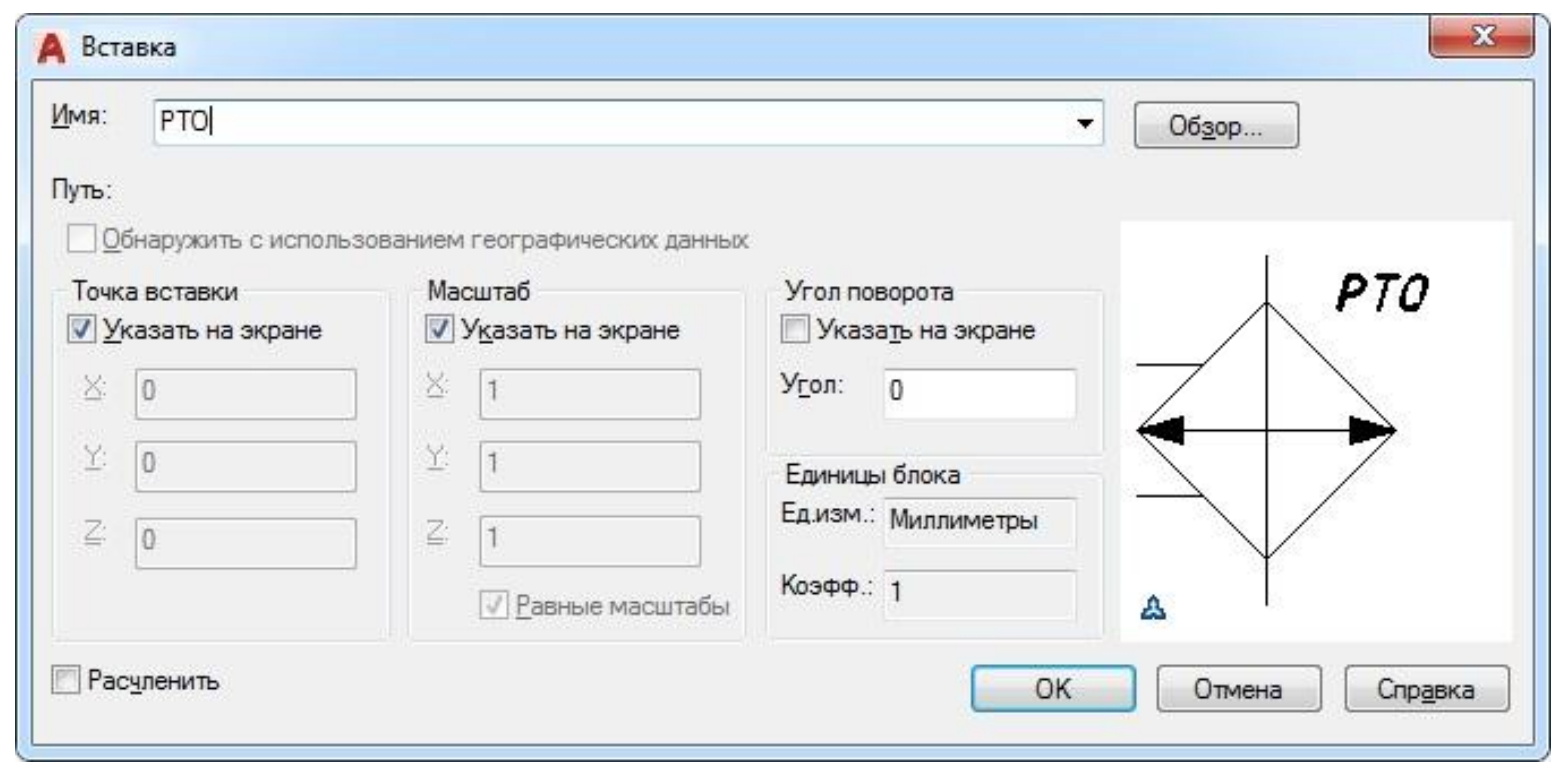

Рисунок 3 - Окно вставки изменяемого блока

В выпадающем меню Утилиты (Tools) выбирается опция Адаптация (Customize), далее - Интерфейс (Interface), благодаря чему открывается окно Адаптация пользовательского интерфейса (Customize User Interface), в нём по умолчанию уже открыта вкладка Адаптация (Customize). Напротив выпадающего списка Aдаптащия: Bce файль (All Commands Only) выбираем кнопку Создание новой команды (Create a new command) (рисунок 4).

При нажатии на кнопку создания новой комманды в правой части окна открываются свойства новой команды. В свойства команды входят: пиктограмма команды, имя, описание, файл справки, название команды на дисплее, макро, метка, идентификатор команды и рисунки для команды (рисунок 5). 


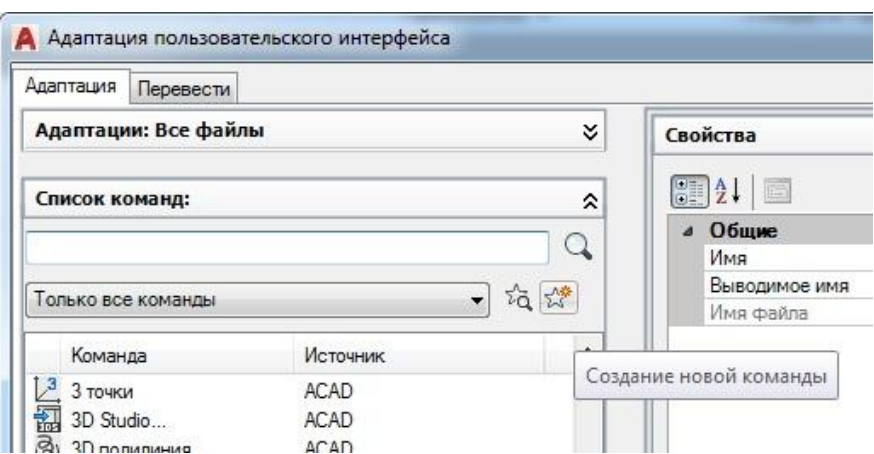

Рисунок 4 - Создание новой команды

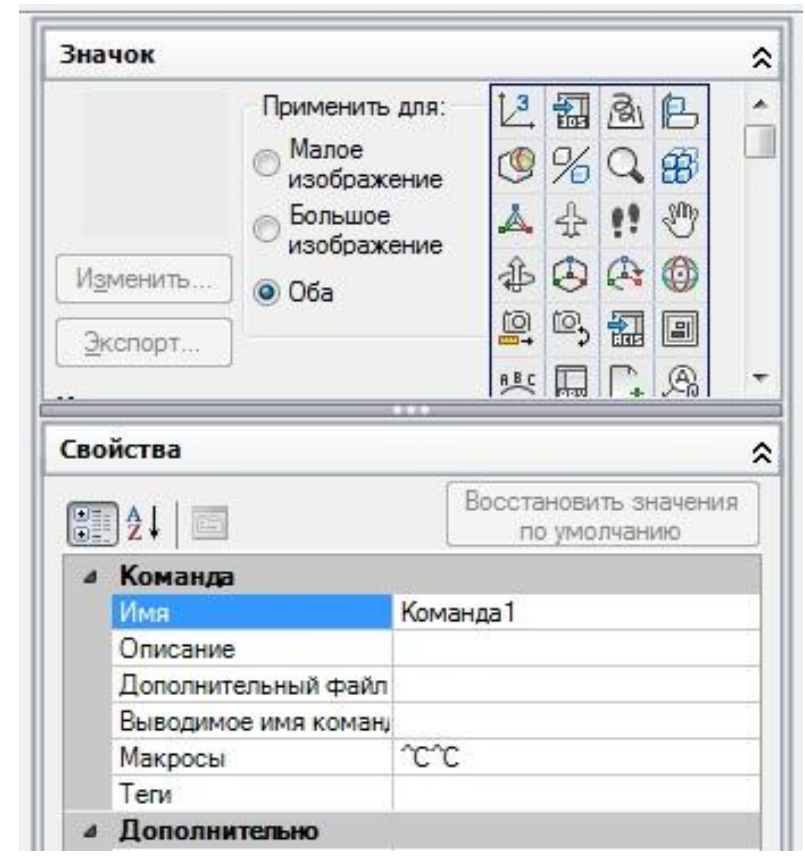

Рисунок 5 - Свойства новой команды

Макрокоманда записывается в строке Maкросы (Macro). По умолчанию там уже есть знак ${ }^{\wedge} \mathrm{C}^{\wedge} \mathrm{C}$, который отвечает за двойное нажатие клавиши ESC. Макрокоманда может быть как короткой, например, команда стереть со свойством всё, так и достаточно длинной. Пример первого варианта на рисунке 6. После записи строки Maкросы (Macro) ставится точка с запятой аналог нажатия на клавиатуре клавиши ENTER. Нижнее подчеркивание применяется в локализованных версиях AutoCAD (например, в русскоязычной). Ставится перед некоторыми командами на английском языке. Приведенные символы используются наиболее часто. Так как процесс выбора параметров для изменяемых блоков в зависимости от их количества может быть достаточно длительным, то можно автоматизировать его с помощью разработки панели команд. Например, команды поворота на 120 градусов.

Во втором случае для удобства записи с помощью троеточия в конце строки Maкросы (Macro) можно открыть окно Редактор длинных строк (Long String Editor) (рисунок 7). Размеры окна можно изменять. Например, довольно популярна запись определённого стиля печати с помощью макрокоманд.

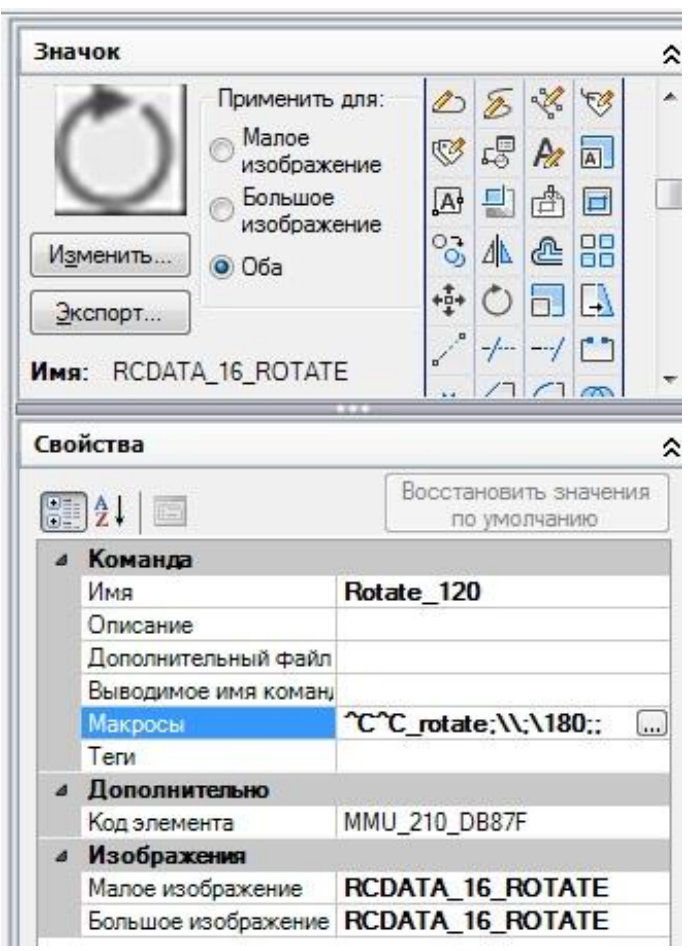

Рисунок 6 - Кнопка «Rotate_120»

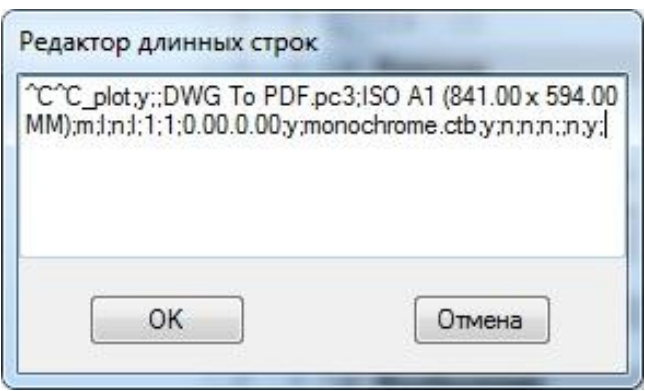

Рисунок 7 - Печать файла с указанными свойствами

После всех записей параметров новой кнопки и её строки Maкросы (Macro) сохраняем все изменения и принимаем (применяем) их. Новую кнопку можно добавить на любую из существующих панелей инструментов или на специально созданную панель инструментов.

Примеры возможного использования: создание собственного набора кнопок, установка указанных настроек, построение и автоматизация изменений объектов по заданным параметрам, автоматизированное построение примитивов по заданным координатам, на более сложном уровне возможность использования языков DIESEL и AutoLISP в макрокомандах.

Преимущества использования: возможность автоматизации работы благодаря указанным изначально свойствам, применение связки команд, что часто используется в определённого рода проектах, доступность при каждой загрузке, возможность использования в облегчённой версии - AutoCAD LT.

Недостатки использования: необходимость изучения специальных символов, применяемых в макрокомандах, детальное знание команд и обозначений их свойств, процесс создания новой кнопки может быть длительным. 
В среде AutoCAD пользователю предоставляется два способа взаимодействия - «кнопочное» через панели команд и «прямое» введение команд в командной строке. Третий из рассмотренных методов автоматизации проектирования работает непосредственно с командной строкой. Он представляет собой использование заранее созданного файла, в котором может находиться: последовательность команд, их опций, команды запуска файлов, открытие инструментальных палитр, то есть, только то, что можно ввести в командной строке. Этот метод автоматизации имеет несколько названий - скрипт, пакет, сценарий. Файл должен иметь расширение .scr и создается из обычного текстового документа с кодировкой ASCII без знаков форматирования. Выбор кодировки обусловлен тем, что она описывает наиболее часто используемые англоязычными пользователями символы - латинские буквы, арабские цифры и знаки препинания. Также в ASCII попадают такие служебные символы как: скобки, кавычки, косая черта и т.п. [5, 6].

Для примера рассмотрим задачу - создание в чертеже $\boldsymbol{n}$-го множества слоёв для размещения в них выносок, таблиц с параметрами, элементов чертежа и т.д. Создание одного слоя включает в себя: имя слоя, тип линии, толщину линии, свойства печати, описание, статус и др. При создании нового слоя необходимо указать имя и цвет слоя, в примере укажем ещё и статус одного из них как рабочего (активного).

Сначала создается новый текстовый файл (например, в текстовом редакторе Блокнот). В файле прописываем _.LAYER - открытие окна слоя, в нём создание нового слоя, на следующей строке имя слоя или имена через запятую, если их несколько. Пробелы между именами не ставятся, так как каждый пробел в файле .scr воспринимается как нажатие клавиши ENTER. Далее указывается параметр - цвет, его значение и имя слоя, которому он присваивается. Так повторяется для каждого слоя (рисунок 8). Перейдем к указанию на слой, который будет рабочим. Для указания этой опции используется её первая буква $-\mathbf{S}$, на следующей строке указывается имя рабочего слоя. После последней строки нужно дважды нажать клавишу ENTER, для выполнения выхода из опции и из самой команды Слой (Layer).

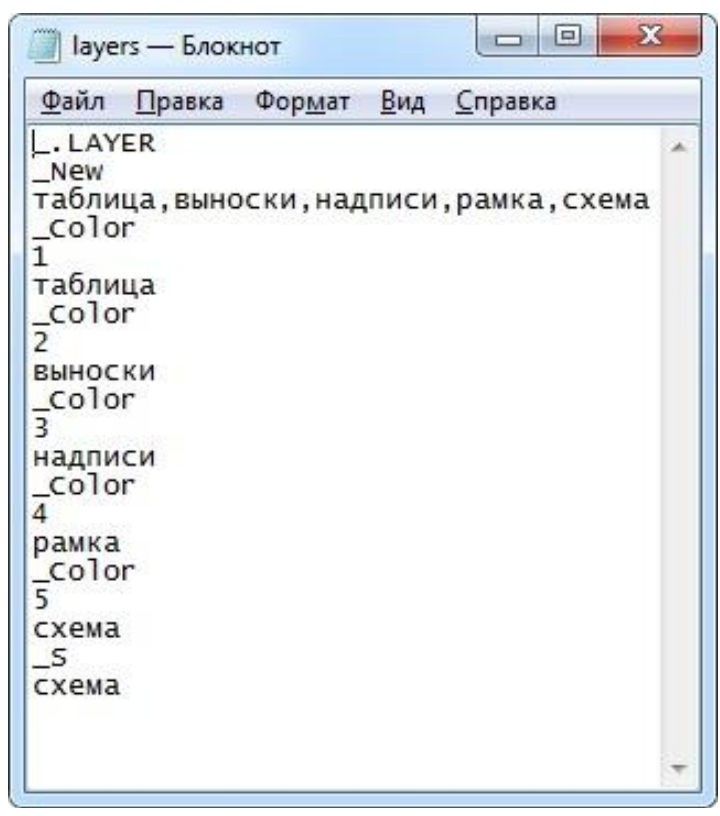

Рисунок 8 - Текстовый файл сценария

При необходимости можно задать для каждого слоя свои опции, собственно, как и количество необходимых для работы слоёв. Подобные файлы могут быть достаточно длинными и подробными. Необходимо проследить, чтобы расширение файла сценария было заменено на .scr.

Для запуска файла его достаточно просто перетащить на рабочее пространство файла чертежа. После загрузки в командной строке отображаются не просто выбранные параметры, но и изначальные запросы программы. Каждый файл типа .scr начинается с команды Скрипт (Script) и указания месторасположения файла (рисунок 9).

Результат выполнения файла layers.scr можно проверить, открыв окно диспетчера слоёв для файла чертежа, на рабочее пространство которого его перемещали (рисунок 10).

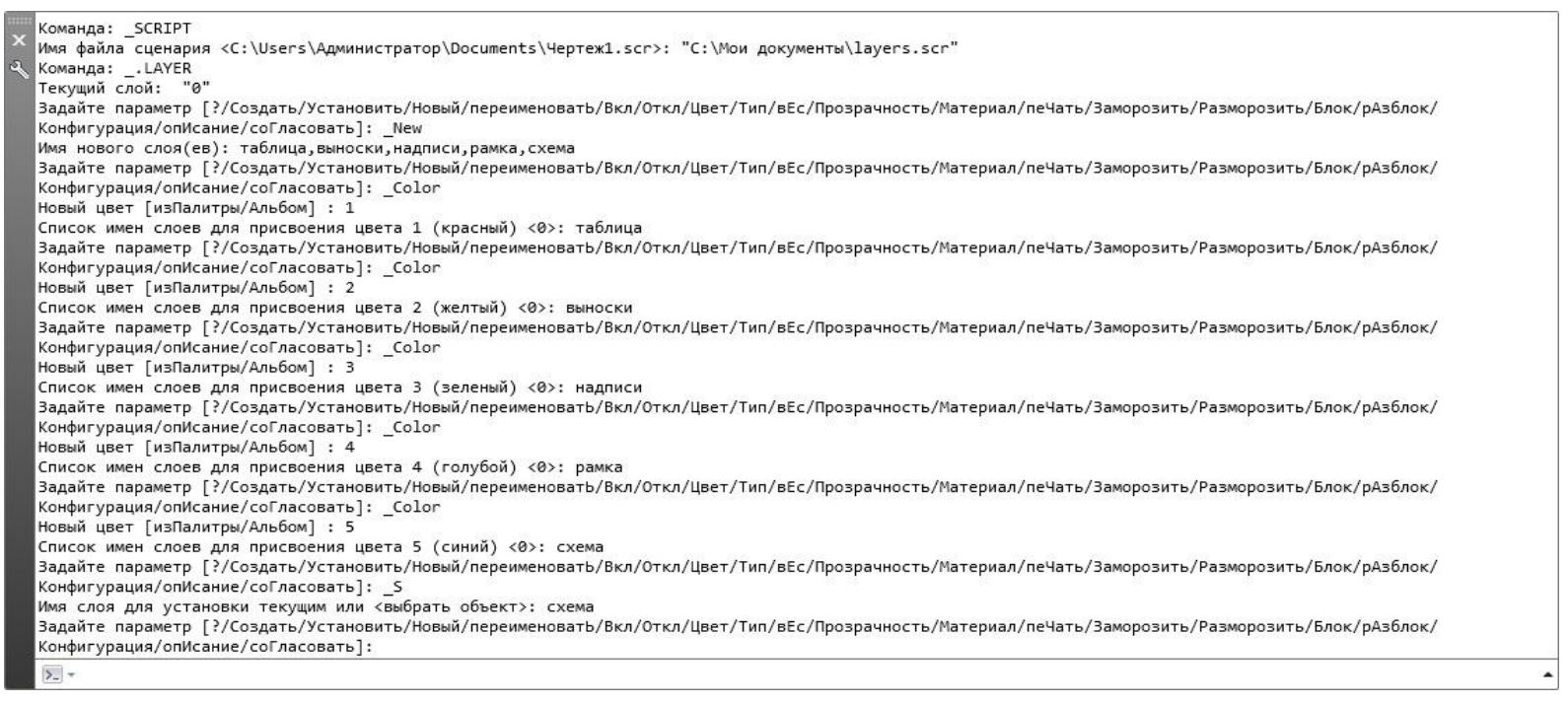

Рисунок 9 - Отображения работы сценария в командной строке 


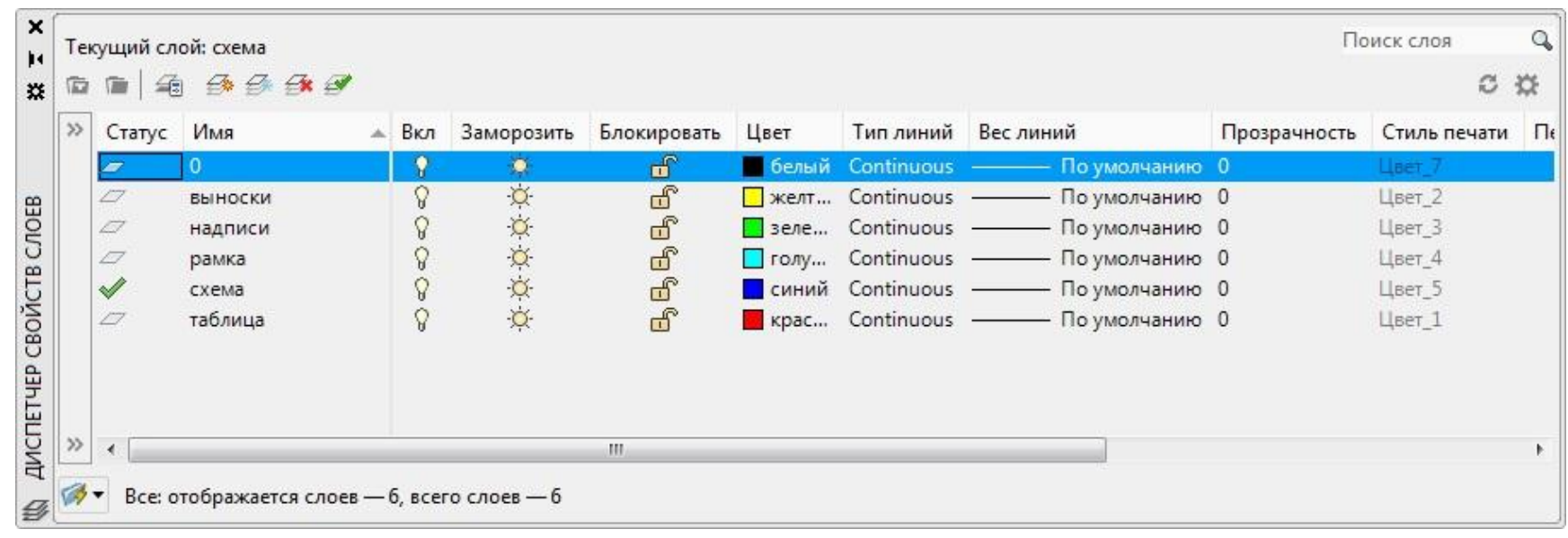

Рисунок 10 - Результат выполнения сценария файлом layers.scr

После выполнения сценария можно присвоить каждому слою свои элементы.

Примеры возможного использования: создание файлов чертежей, размерных стилей и т.п., изменение настроек, редактирование элементов чертежа, запуск других сценариев, изменение параметров листов, запуск печати по сценарию для чертежей определённого типа и др.

Преимущества использования: возможность использования в облегчённой версии - AutoCAD LT (в полной версии с помощью сценариев можно использовать команды и функции, описанные при помощи AutoLISP, ARX или VBA); возможность автоматического выполнения при запуске AutoCAD; возможность разработки сценариев с наборами свойств для разных типов файлов, чертежей и документов.

Недостатки использования: нет возможности установки значения переменных по типу файлов AutoLISP (только запуск файлов AutoLISP); после запуска сценария его нельзя поставить на паузу, а только остановить или перезапустить; в случае ошибки сценарий останавливается в ожидании действий пользователя либо может работать некорректно.

\section{3 Выводы}

Рассмотренные методы автоматизации проектирования в среде AutoCAD доступны любому пользователю, не требуют навыков программирования и использования дополнительных программ, могут использоваться в разных версиях AutoCAD и AutoCAD LT, включая специализированные версии, значительно уменьшают затраченное на работу время и упрощают сам рабочий процесс. Каждый из методов автоматизации предполагает определённые знания, может использоваться для выполнения ряда конкретных задач и имеет свои достоинства и недостатки. Блоки являются со- хранёнными элементами чертежа, для создания которых достаточно только навыков проектирования. Макрокоманды предполагают наличие навыков по работе со свойствами и настройками для панелей и кнопок AutoCAD, знание спецсимволов макрокоманд, команд AutoCAD и их свойств. Сценарии для написания требуют знаний команд, командных опций, специальных функций, применяемых для пакетов, то есть всё, что возможно вводить в командной строке. Таким образом, в среде AutoCAD существует множество встроенных возможностей для его адаптации к решению конкретных задач в области холодильной техники и автоматизации рабочего процесса.

\section{Литература}

1. Omura, G., Benton, B.C. Mastering AutoCAD 2018 and AutoCAD LT 2018. - Sybex, 2017. - 1080 p.: ISBN13 978-1119386797

2. Габидулин В. Адаптация AutoCAD под стандарты предприятия. - М.: ДМК пресс, 2016. - 210 с.: ISBN 978-5-97060-239-3

3. Зуев С.А., Полещук Н.Н. САПР на базе AutoCAD как это делается. - СПб.: БХВ-Петербург, 2004. 1168 c.: ISBN 5-94157-344-8

4. Свет В.Л. AutoCAD: язык макрокоманд и создание кнопок. - СПб.: БХВ-Петербург, 2004. - 320 с.: ISBN 594157-392-8

5. Тику, Шам. Настройка AutoCAD. - М.: ИД «Вильямс», 2006. - 768 с.: ISBN 5-8459-0931-7, 1-4018-5135-5

6. Эбботт, Д. AutoCAD: секреты, которые должен знать каждый пользователь. - СПб.: БХВ-Петербург, 2008. - 640 c.: ISBN 978-5-9775-0214-6

Отримана в редакції 04.05.2017, прийнята до друку 06.06.2017 


\section{Automation Methods of Design in AutoCAD Environment}

\section{P. B. Lomovtsev, S. V. Boltach, N. F. Mitrofanova, B. G. Shinko}

Odessa National Academy of Food Technologies, 112, Kanatna Street, city of Odessa, 65039, Ukraine

Creating large-scale or highly specialized projects in the AutoCAD environment takes a lot of time. To reduce design time and add functionality, various methods of design automation are used. Most of them, besides the knowledge of commands and the interface of the AutoCAD environment, require skills in programming. The methods of design automation for the field of refrigeration, which require only knowledge of work in the AutoCAD environment, are considered in this paper. Each method is analyzed; examples of their use and application are given. Advantages and disadvantages are indicated.

Keywords: Design Automation Methods; AutoCAD; Schematic Diagram; Block; Library of Elements; Scenario; Macrocommand.

\section{References}

1. Omura, G., Benton, B.C. (2017) Mastering AutoCAD 2018 and AutoCAD LT 2018. Sybex, 1080 p. ISBN-13 9781119386797

2. Habydulyn V. (2016) Adaptaciia AutoCAD pod standarty predpriiatiia. Moscow, DMK press, 210 p. ISBN 978-5-97060-239-3

3. Zuev S.A., Poleshhuk N.N. (2004) SAPR na baze AutoCAD - kak эto delaetsia. BHV-Peterburh, 1168 p. ISBN 5-94157-344-8
4. Svet V.L. (2004) AutoCAD: Iazyk makrokomand i sozdanie knopok. BHV-Peterburh, 320 p. ISBN 5-94157-3928

5. Tyku, Sham. (2006) Nastroika AutoCAD. ID Wilyams, 768 p. ISBN 5-8459-0931-7, 1-4018-5135-5

6. Эbbott, D. (2008) AutoCAD: sekrety, kotoryie dolzhen znat kazhdyi polzovatel. BHV-Peterburh, 640 p. ISBN 9785-9775-0214-6 